\title{
EELS of the Interface between Al and Glass Formed with a High Speed Collision Process of Cold Spray
}

\author{
Minghui Song ${ }^{1}$, Hiroshi Araki ${ }^{2}$, Seiji Kuroda ${ }^{2}$ and Kazuhiko Sakaki ${ }^{3}$ \\ 1. TEM Station, National Institute for Materials Science (NIMS), Tsukuba, Japan. \\ 2. Research Center for Structural Materials, NIMS, Tsukuba, Japan. \\ 3. Faculty Eng., Shinshu University, Nagano City, Japan.
}

Cold spray (CS) uses a high pressure gas to accelerate powder particles onto a substrate to form coatings at temperatures much lower than the melting point of the sprayed particles. For its low working temperature and flexibility in applications, CS process has been researched extensively and used increasingly in industries for various coatings including the coating of metals on a ceramic or a glass substrate. One of the examples is the application in fabrication of power-module, where metal coatings of $\mathrm{Al} / \mathrm{Cu}$ are formed on an alumina substrate as a heat-sink [1]. However, the bonding mechanisms of metals on ceramic or glass substrates have not been clarified yet. Experimentally, Al shows excellent bonding onto glass or some ceramic substrates, while $\mathrm{Cu}$, Ti, and some other metals, do not [1,2]. Chemical properties of the sprayed metal and substrate seem to play important role in the bonding. Detailed characterization of the bonding interface with TEM is helpful to understand the nature of the bonding. It has been reported that there is an interface layer formed due to interaction between the CS Al and glass [3]. In the present work, transmission electron microscopy observation and analysis, especially the electron energy loss spectroscopy (EELS) analysis were carried out for the bonding interface of $\mathrm{Al} /$ glass, to investigate the distribution of elements and chemical status of the interface layer in detail.

CS was performed using a spray system with a converging-diverging barrel nozzle. Al powder with purity of 99.85 mass \%, and an average size of $17.3 \mu \mathrm{m}$, was used. Soda-lime glass plates in size of 76 $\mathrm{mm}(\mathrm{L}) \times 26 \mathrm{~mm}(\mathrm{~W}) \times 1.2 \sim 1.5 \mathrm{~mm}(\mathrm{~T})$ were used as the substrate. Singly impacted Al particles were obtained on the substrate with CS [3]. Cross-sectional TEM specimens were prepared using an FIB apparatus, JEM-9320FIB (JEOL Ltd.). The TEM observations and EELS analysis were performed using JEM-2100F (JEOL Ltd.).

Figure 1 shows the typical results for a $20 \mu \mathrm{m} \mathrm{CS} \mathrm{Al} \mathrm{particle} \mathrm{on} \mathrm{a} \mathrm{glass} \mathrm{substrate.} \mathrm{The} \mathrm{interface} \mathrm{layer}$ (interaction layer) is observed in a thickness of $\sim 80 \mathrm{~nm}$ in the center region of the Al particle (Figure 1a). Figure $1 \mathrm{~b}$ and $1 \mathrm{c}$ are STEM HAADF images of the interface layer near the center of the particle. 3 sublayers are identified in the interface layer, counted as 1,2 and 3 from the glass substrate hereafter (SL1, SL2, and SL3). The thickness of the sublayer is not the same everywhere, especially SL1. The contrast (Z-contrast) of the sublayers implied that SL1 and SL3 have the similar density, but SL2 has a lower density. The existence of the low density layer has been considered to be attributed to a tensile stress between a CS particle and the substrate during a particle collision process [4]. EELS analysis was conducted crossing the interface layer along line A and B indicated in Figure 1c. Core loss edges of Al$\mathrm{K}$ and $\mathrm{Si}-\mathrm{K}$ were analyzed along line $\mathrm{A}$. The profile along the line is shown in Figure 1d. Si-K edge was detected in the glass substrate with a chemical shift corresponding to $\mathrm{Si}$ in $\mathrm{SiO}_{2}$, but not detected in the interface layer. On the other hand, Al-K edge with a chemical shift similar to that of Al in Al-O bonds was detected in the interface layer, though the intensity of signals is weaker in SL2 than in SL1 and SL3. This may be mainly due to the lower density of SL2. The profile of O-K edge measured along line B is given in Figure 1e. O-K edge with chemical shift was detected in all the 3 sublayers. In addition to the 
main part of $\mathrm{O}-\mathrm{K}$ edge, in which the first peak is at $\sim 539 \mathrm{eV}$, there is a pre-O-K peak with the peak at $\sim 531 \mathrm{eV}$, apparently in SL1 and SL3. By referring the literature [5], it is considered that the pre-peak may be resulted from $\mathrm{O}-\mathrm{O}$ bonds in the interface layer. The $\mathrm{O}-\mathrm{O}$ may exist in the interface layer because the reaction layer was formed in a highly non-equilibrium condition in a CS process. Weak signals of $\mathrm{Na}$ and $\mathrm{Ca}$ were detected in SL2, but only $\mathrm{Al}$ and $\mathrm{O}$ were detected in SL1 and SL3. Therefore, it was revealed that the interface layer is mainly composed of $\mathrm{O}$ and $\mathrm{Al}$, but with almost no $\mathrm{Si}$. These results suggest that the interaction between the CS Al and the glass substrate did not processed by mixture of $\mathrm{Al}$ and glass, but by reaction of $\mathrm{Al}$ with $\mathrm{O}$ at the surface of the glass substrate. This is believed to be an important hint for understanding the bonding between the sprayed Al and glass substrate, as well as the bonding between other metals and glass or ceramics formed with CS.

\section{References:}

[1] K-R Donner, F Gaertner, and T Klassen, J. Therm. Spray Technol. 20 (2011), p. 299.

[2] K Sakaki et al, Proc. the $18^{\text {th }}$ Meeting on Materials and Process of The Japan Society of Mechanical Engineers (2010), p. 438.

[3] M Song et al, J. Phys. D: Appl. Phys. 46 (2013), p. 195301.

[4] K H Kim, M Watanabe, S. Kuroda, J. Therm. Spray Technol. 18 (2009), p. 490.

[5] N Jiang, J C H Spence, Ultramicroscopy 106 (2006), p. 215.

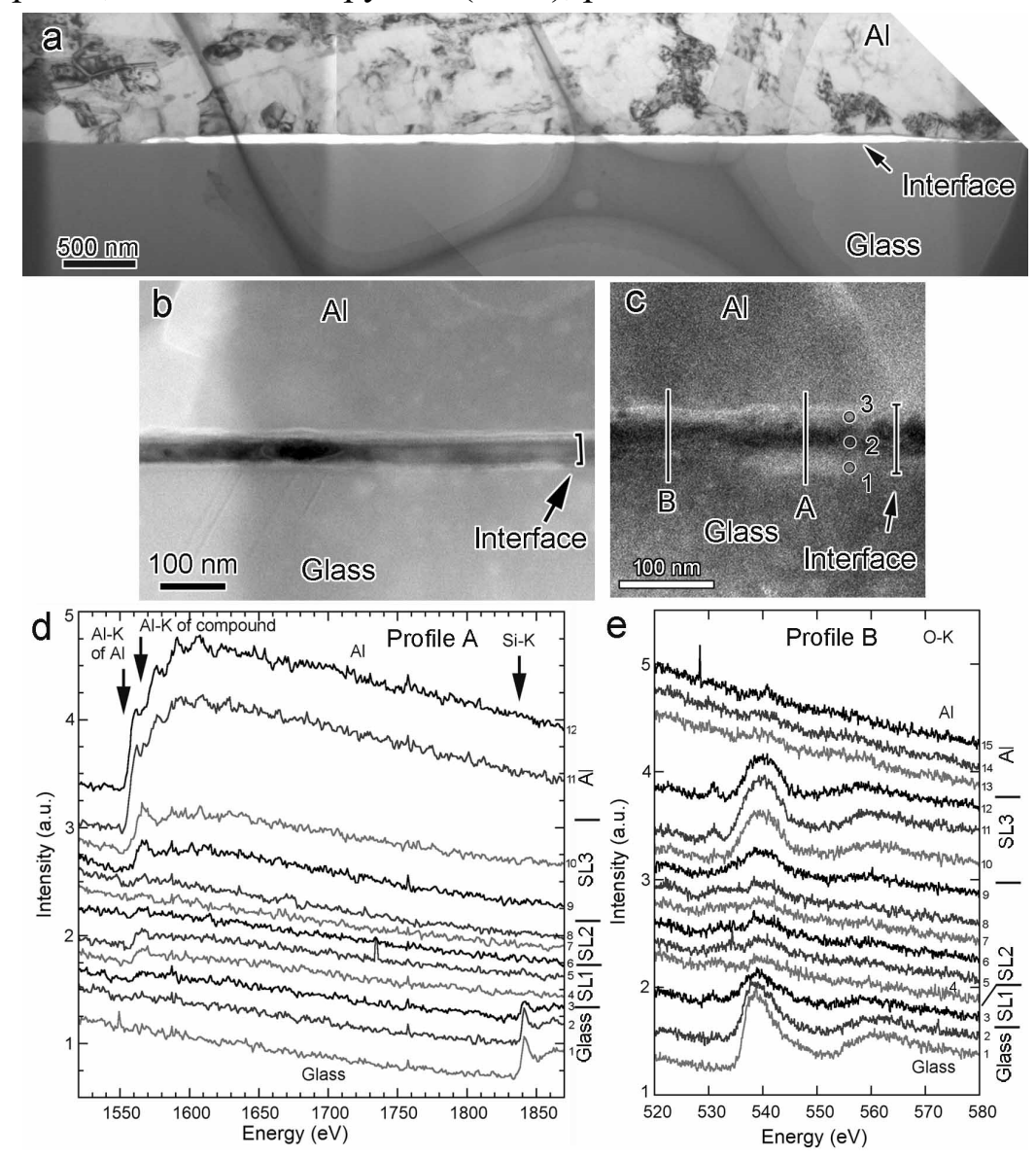

Figure 1. TEM (a) and STEM-HAADF (b, c) images of a cross-sectional specimen of the bonding interface between a $20 \mu \mathrm{m} \mathrm{Al}$ particle and a glass substrate formed with cold spray. (d and e) EELS spectra profiles measured along line A and B indicated in (c), respectively. 1, 2, and 3 in (c) indicate sublayer 1 to 3 (SL1, SL2, and SL3) in the interface layer, respectively. 